\title{
PERIPHERAL BRACHIAL PARALYSIS IN INFANTS AND CHILDREN *
}

\author{
BY
}

\author{
J. EDGAR MORISON, M.B., B.Sc.
}

(From the Belfast Hospital for Sick Children, Belfast, Northern Ireland)

In 1861 Duchenne noted in four cases of ' subacromial subluxation,' thought to be produced at birth, a true paralysis of the brachial plexus. In 1872 he described in detail obstetrical paralysis, with or without fracture or subluxation, and in older children described subspinous luxation as complicating or producing the paralysis (Duchenne, 1872). Erb, in 1874, localized by faradic stimulation an area in the neck associated with the contraction of the muscles most commonly paralysed in this lesion. This point was later associated with lesions at the junction of the fifth and sixth cervical roots (reviews by Williamson, 1897 ; Kennedy, 1903). Whereas the earlier observers blamed direct pressure from forceps, the midwife's finger or the subject's clavicle, the work of Fieux and more especially Duval and Guillain towards the end of the last century emphasized traction on and rupture of the upper roots of the plexus from forcible separation of the head and shoulder. Much experimental work on the cadaver has supported this view (Delbet and Cauchoix, 1910 ; Sever, 1920 ; Taylor, 1921). Whitman (1905) by his work on reduction of the subluxation sometimes present emphasized this aspect, and several workers have followed T. Turner Thomas (1911) in regarding the subluxation as primary in some cases at least (Ashhurst, 1918 ; Lange, quoted from Thomas, 1916 ; and Platt, 1921).

\section{Case records}

The relevant cases attending the Belfast Hospital for Sick Children for the past six years have been investigated. These twenty-nine cases, which include three post-natal traumatic lesions, are entirely unselected and may be considered quite representative. Though the number of cases is small a study of the literature suggests that it falls to the lot of few to examine a large number Fairbank (1913) reported forty cases, Froelich (1921) and Gilmour (1925) each encountered twenty-five cases, and McFadden (1928) studied fifteen cases. Sever (1925) of Boston has, however, collected data of eleven hundred cases. An analysis of this material illustrates the almost infinite variation which may be met with in the evolution of this type of lesion, and how artificial is any attempt to describe the later development of these children in a few general classes, however similar many may appear when first presented. A summary of a number of selected cases will illustrate the variation in recovery and function attained.

- Based upon a thesis awarded the Davis Memorial Scholarship, 1938. 
Case 1. A full-time female child was born as a vertex to a primipara after a twelve-hour labour with anaesthesia and instruments. The child showed no asphyxia and no bruising. When first seen at the age of three weeks she showed a right-sided sterno-mastoid swelling, which increased for a few weeks, but gradually disappeared. The right arm hung vertically from the shoulder, internally rotated, fully extended and pronated at elbow. There was no carpal deviation, no definite wrist drop and, though the digital muscles appeared normal, the fingers were flexed and thumb adducted (fig. 1). There was no tenderness and no resistance to passive movement. The most powerful displacing forces were the pectoralis major and the latissimus dorsi muscles. The

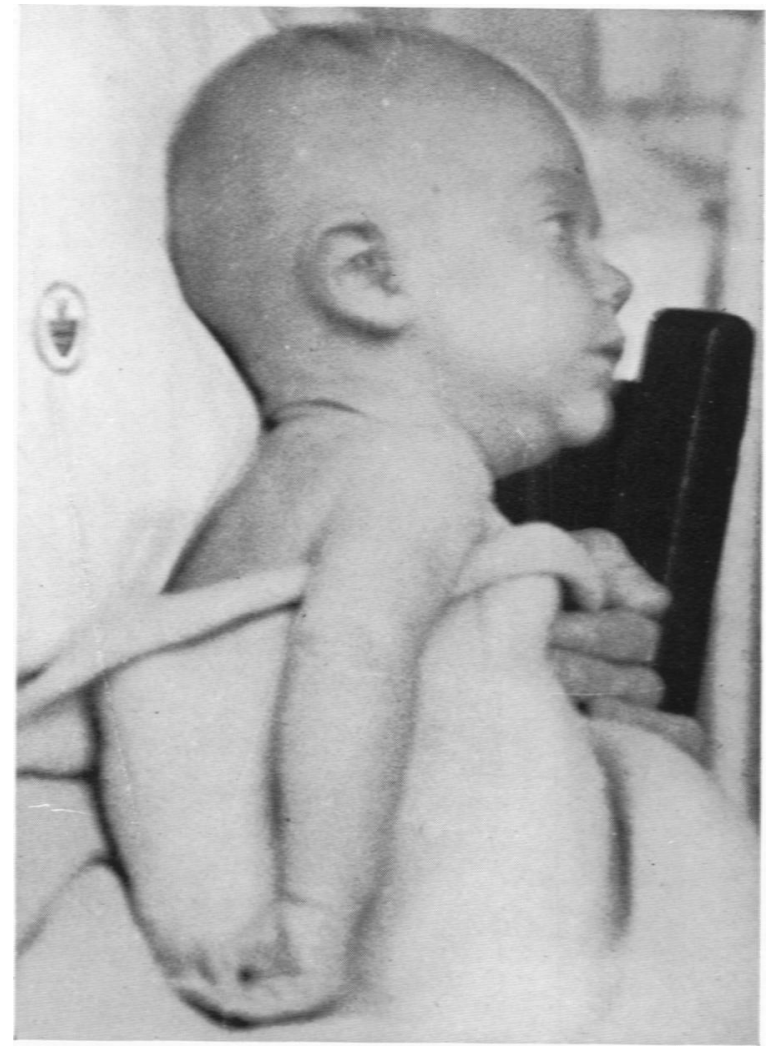

Fig. 1.-The earliest position of the limb.

paralysed muscles were those typically involved in the upper arm type of birth palsy.

Treatment by a right angle abduction splint relaxed the paralysed muscles and movements and light massage were given. After three months supination at the elbow was slightly better and wrist drop could be definitely excluded, the humeral head was very mobile and there was slight wasting of the deltoid, spinati and elbow flexors, but no contractures in the active muscles. After five months the elbow flexors, though weak, had recovered some power. As evidence of recovery in the shoulder muscles the deltoid was beginning to ' fix' the shoulder for abduction by scapular movement. Six months later recovery was still incomplete at the shoulder, but no contractures had developed. 
This patient was treated early with delay in recovery. The value of treatment is shown by the control exercised on the development of contractures. Another similar example was seen showing better, but not complete, recovery after six months.

Case 2. This was a female infant, a fifth child, since followed by four normal children, all easy births. She was a large full-time child born under anaesthesia as a breech five to six hours after rupture of membranes. The obstetrician thought he heard something give a crack during delivery. No other injuries were noted, but the baby was difficult to resuscitate. The left arm and forearm hung limp from birth, the wrist and hand were normal. The infant was treated for six weeks from first week by fixation of arm behind head by collar and cuff and without massage. Some improvement was noted in three weeks. The baby started to move whole arm in six weeks.

On examination at age of five years she is a healthy, well-developed child. Shoulder and arm are perfectly normal in appearance. The arm swings spontaneously when walking. All passive and active movements are full. When abducting the arm from the side movement starts in the deltoid arc: after about $45^{\circ}$ abduction the inferior angle of the scapula, which has remained relatively steady, glides outwards. Range and strength of abduction and external rotation are equal on both sides.

Five other similar cases were observed. Two, treated with arm in sling in front of body, a bad position, showed complete recovery, as did a case treated by massage only.

Case 3. A first child, a female, was born as a vertex with instruments after thirty-six hours. There was much difficulty in delivery of shoulders and later in starting respiration. The ear and face were cut and a right sternomastoid tumour developed later. In addition to typical position of the arm, the right scapula was always prominent. She was treated from fifth week by fixation of hand by bandage behind head in correct position to relax all paralysed muscles, and from sixth to fifteenth month in an aeroplane splint. Slight recovery was noted at eighth week and shoulder abduction at seventh month.

When examined at age of two years she showed slight prominence of both scapulae at rest, and marked winging of right when arm abducted, especially when pushing against resistance. The right arm could be elevated just above the horizontal (right serratus magnus weakness, not paralysis). Deltoid recovery was good, and external rotation fair. The hand in a useful supinated position could reach the mouth. The arm was used well and there was no wasting nor shortening.

X-RAY : Bony outlines normal. Normal diaphragmatic level.

This patient is recovering satisfactorily, and without disabling contractures. Serratus magnus paralysis is present, but now only residual.

Case 4. After an instrumental delivery a female infant, said to weigh $14 \mathrm{lb}$., was born to a multipara five hours after rupture of the membranes. The right arm hung limp, but fingers and hand could be moved from the beginning and there were no other birth injuries. She was treated for six months by suitable fixation and by massage and movements for a further year.

Now the child aged five years shows the right arm held in marked internal rotation and slight forward flexion at shoulder, with about $20^{\circ}$ flexion and full pronation at elbow. There is slight wasting over tip of shoulder, and some generalized atrophy of hand with shortening of $\frac{1}{2}$ in. in the arm and less than 
$\frac{1}{2}$ in. in the forearm. Bony points are normal, but there is definite limitation of passive external rotation $\left(45^{\circ}\right)$, while passive abduction is almost full. The deltoid can fix the shoulder and move the arm slightly only with minimal load. Normally abduction is by the scapula, and the hand can be raised only to level of chin, while active external rotation is deficient. At the elbow there is deficient full passive extension, and about half full supination. Active extension and flexion at the elbow is functionally satisfactory, but the deficient supination and the internal rotation of the arm do not allow the palm of the hand to be turned upwards. There is no actual paralysis of the forearm or

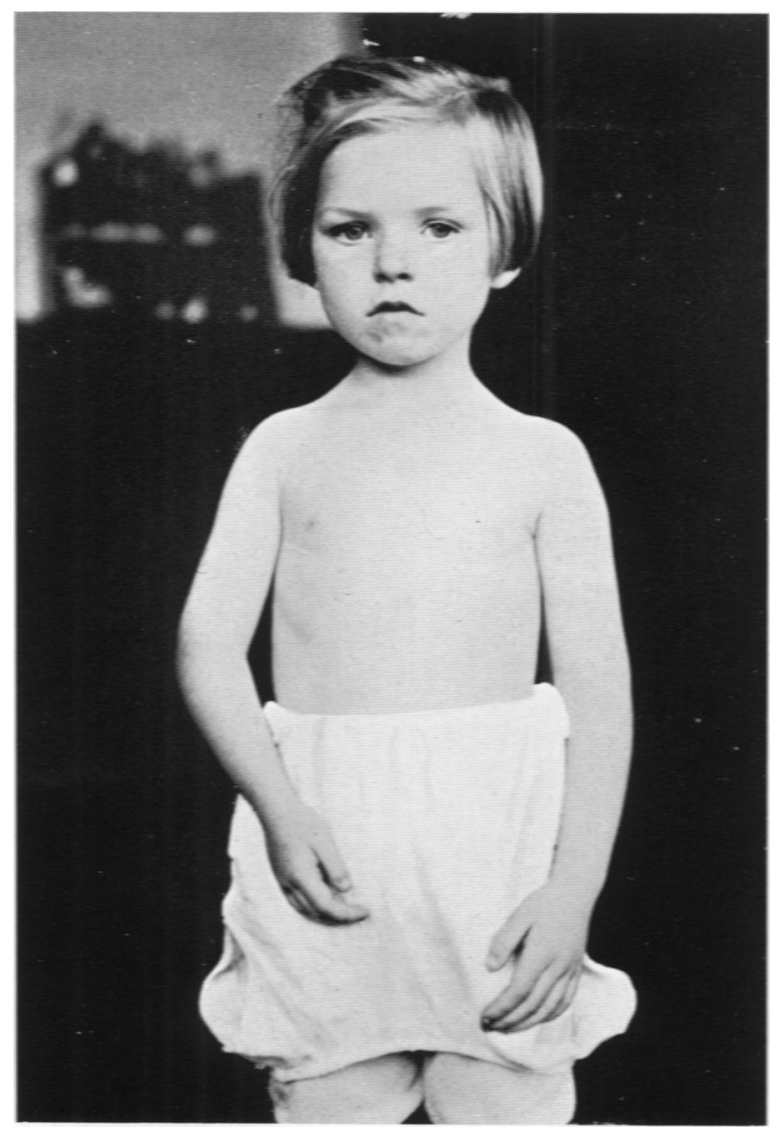

Fig. 2.-Case 5. The effect of disuse.

hand muscles, but with insufficient function there is considerable atrophy of hand with loss of skin creases; nails and finger pulps normal.

X-RAY : Small cervical ribs both sides.

This patient received fairly adequate treatment and recovery is disappointing owing rather to muscle weakness than to contractures. Muscle involvement is not more extensive than usual, though some pre-fixation of plexus is suggested by cervical ribs.

Case 5. A female infant three months old was brought to hospital with the right arm limp following a difficult birth. When four months old, subscapularis 
tenotomy was performed and followed by supervised massage and movements for three months.

Examination at the age of six years shows a normal shoulder outline, no internal rotation of the arm, slight flexion at the elbow, marked wasting over the extensors, right arm shorter than left by $\frac{1}{2}$ in., forearm by $1 \frac{1}{4}$ in. (figs. 2 and 3 ),

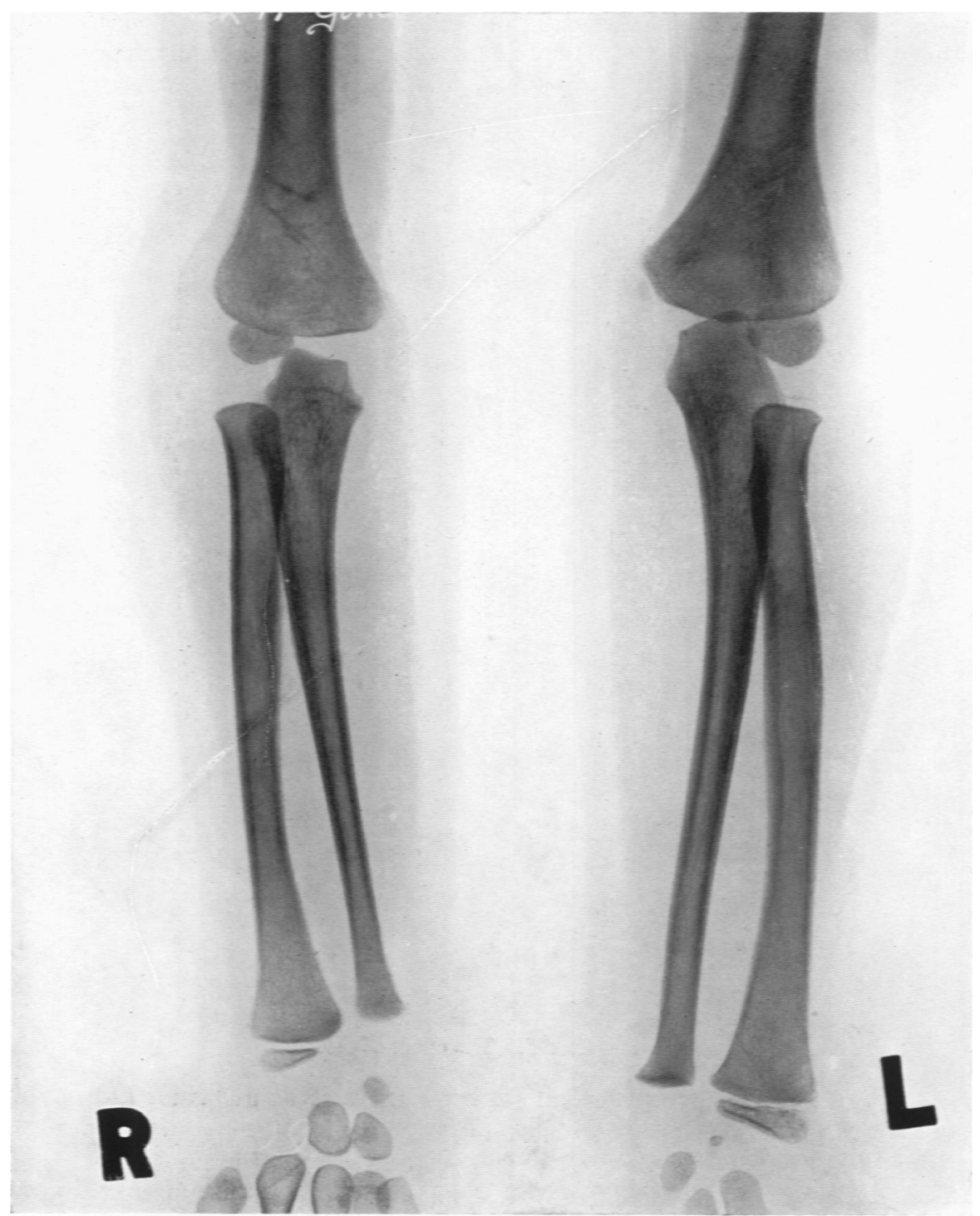

Fig. 3.-Case 5. Atrophy of forearm bones.

slight ulnar carpal deviation and wrist drop in rest position. There is less than $45^{\circ}$ limitation of active external rotation at shoulder, and definite limitation of abduction which occurs by scapular movement unless with minimal load. The main residual weakness is in carpal extensors-in grasping dorsi-flexion does 
not occur and there is slight abduction weakness of thumb. The hand is poorly developed, but all intrinsic muscles are normal.

This patient shows less disability than usual at shoulder and recovery of external rotation better than true abduction. Persistence of wrist drop is no evidence of whole arm lesion. The better explanation is that the carpal extensors are, at least, sometimes supplied by fifth and sixth (Harris, 1904) or even fifth cervical root (Sherren, 1906).

Case 6. A male, full time and the fourth child of an otherwise normal family, was born after a difficult labour and after internal version for a transverse

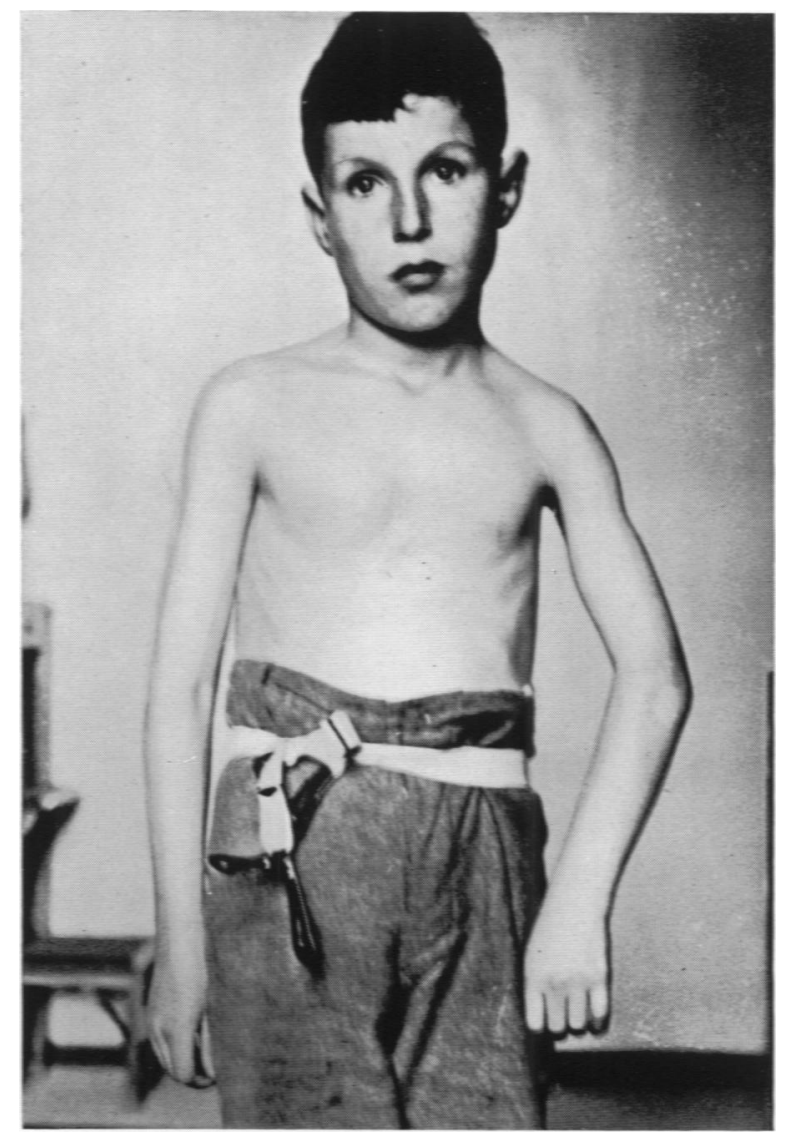

Fig. 4.-Case 6. Marked contractures and subluxation of shoulder joint.

presentation. The left arm was limp, but there were no other birth injuries and no asphyxia. He received no treatment until he first attended hospital at the age of seven years, when an attempt was made to improve position of forearm by excision of the head of the radius.

At the age of ten years he is a healthy, active boy of good nutrition showing definite flattening in front of left shoulder, some wasting of deltoid and spinati, the arm fixed in abduction $30^{\circ}$ from the side, in slight forward flexion and gross internal rotation (fig. 4). The humeral head can easily be felt displaced back- 
wards. There are gross contractures round the shoulder, visibly of the pectoralis major and latissimus dorsi, while only about $30^{\circ}$ passive abduction and less external rotation is possible. Scapular movement is full ; the deltoid just contracts and there is no active external rotation. The forearm is fixed in full pronation, elbow extension is $30^{\circ}$ short of full, but elbow flexors and extensors contract well. The fully pronated hand can be brought up to the level of the chin (fig. 5). Relative shortening-arm $\frac{3}{4}$ in., forearm $\frac{1}{2}$ in. There is no sensory loss.

Skiagrams show cone-shaped humeral head, arthritic changes, and elongation of the coracoid process (fig. 6).

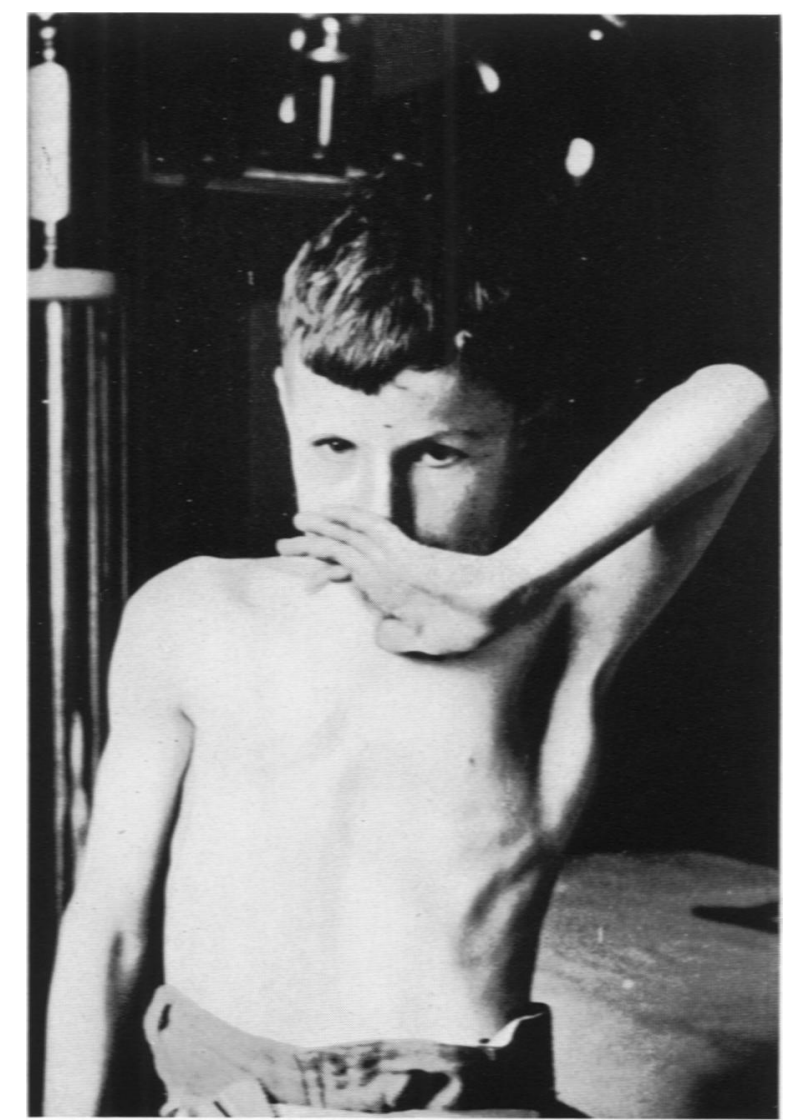

Fig. 5.-Case 6. To show the disability of internal rotation of arm and pronation of forearm.

There is practically no scapulo-humeral movement. The condition is worse than a bony ankylosis, as internal rotation is disabling. To this there is added gross pronation. The patient is an energetic boy who persists in using the limb, which shows comparatively little wasting.

Case 7. She is the sister of case 4, born after a difficult instrumental vertex birth, fifth child, full time. She has received no treatment, except massage and 
movements for one year when five years old. There has been a gradual slight improvement in function, but none noticed during the past two years.

General examination at the age of ten years is negative, without evidence of spinal cord or sympathetic injury, respiratory system normal. There is marked wasting of right arm and chest (clavicle $\frac{3}{4}$ in., arm 3 in., and forearm $1 \frac{1}{2}$ in. relative shortening). The right arm hangs abducted in slight forward flexion, not rotated. The forearm is flexed at the elbow $60^{\circ}$ from full extension and fully supinated. The wrist is in dorsi-flexion and slightly deviated radially. The metacarpo-phalangeal and inter-phalangeal joints are flexed (fig. 7). Contractures limit extremes of passive shoulder joint movement and extension to within $45^{\circ}$ of full at elbow, and permit only $45^{\circ}$ passive pronation at elbow.

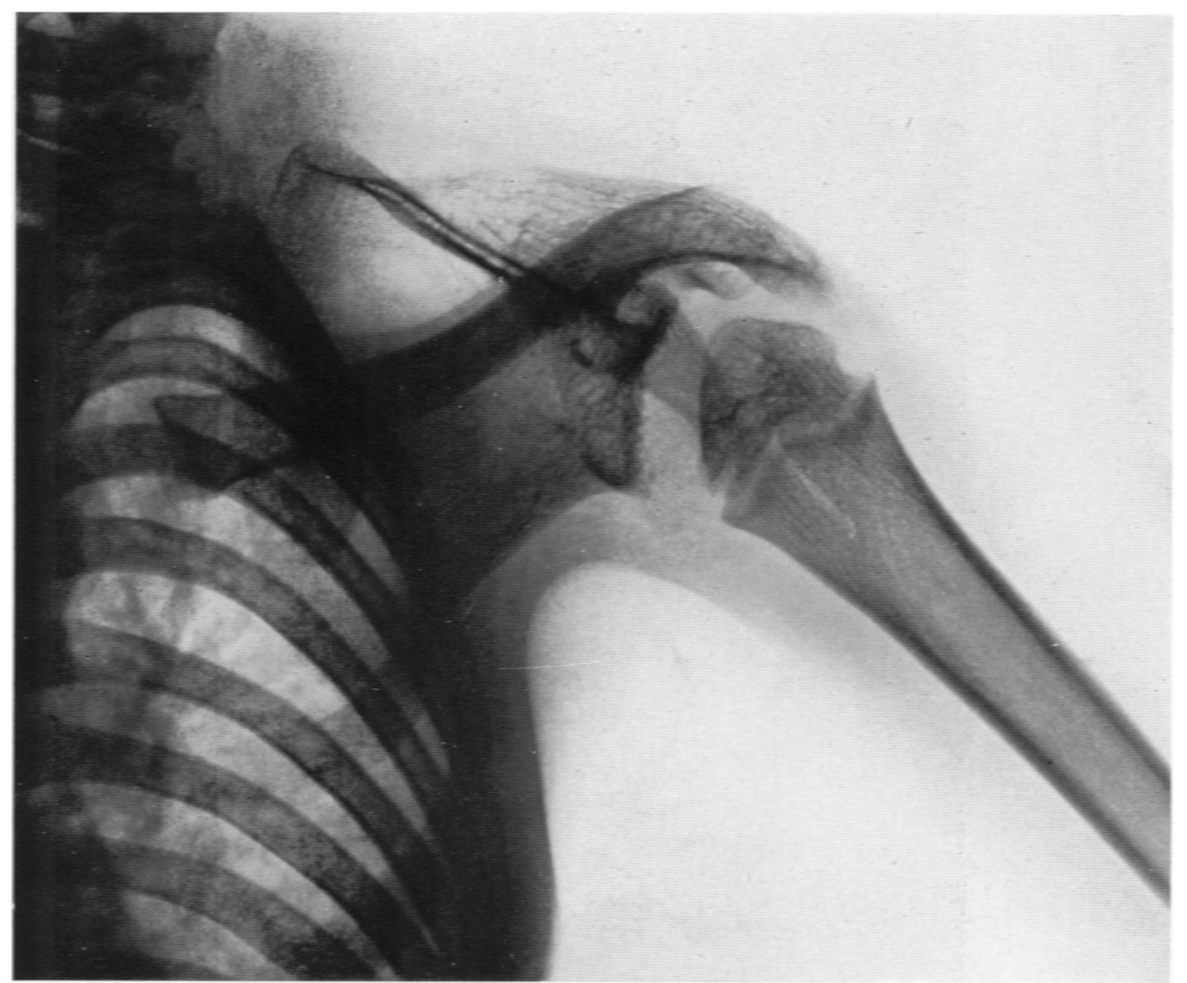

Fig. 6. Case 6. Cone-shaped humeral head, flattened glenoid and elongated coracoid.

The shoulder joint is not flail, but is only sufficiently fixed for about $30^{\text {= weak }}$ abduction by scapula, the muscles acting on which are normal. Movement at the elbow is limited to $45^{\circ}$ weak flexion, and at the wrist to flexion and extension without load. Efforts at grasping are weak, as the fingers cannot close into palm of hand. The limb can steady an object, but no weight can be carried, nor can the hand be elevated above the level of the breast. There is an area on the ulnar side of the forearm back and front, from one inch below the elbow to the wrist, over which sensation to light touch and to pin prick are lost equally. The flare response (Lewis, 1927) is badly marked generally, but appears to be present over this area. Though the skin is thin and devoid of fine hairs, especially over the analgesic area, there is no abnormality of the nails or fingertips such as might be considered a 'trophic' change. 
X-RAY : Shortening and rarefaction of bone, but no deformity of humeral head. Some lengthening of cervical lateral processes of seventh cervical vertebra.

This patient shows whole-arm involvement with slight recovery, chiefly of the upper roots. The paralysis at the shoulder is balanced and there are no contractures. Sensory loss suggests complete lesion of sensory fibres to the

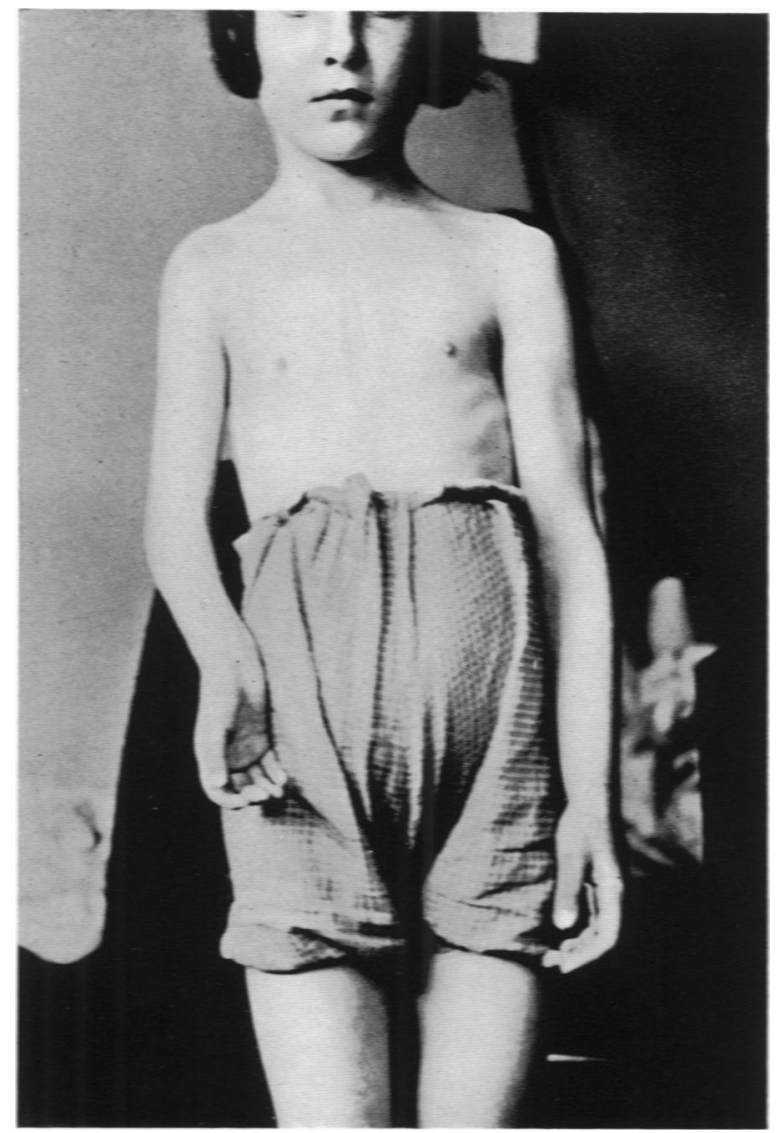

Fig. 7.-Case 7. The limb in a whole arm lesion.

affected area from eighth cervical and first thoracic roots, and the flare response suggests a lesion central to the ganglion.

Case 8. In a railway accident a boy of eleven sustained a compound fracture of the right femur and a fracture of the right humerus, while the left arm appeared painful on movement. He was acutely ill for some time and the left arm, which was radiologically normal, was allowed to lie across the chest, the elbow flexed and pronated.

External rotation or abduction at shoulder or handling the limb, especially pressure over the extensor muscles arising from lateral humeral condyle, produced severe pain and muscle guarding. This was well marked at the end of 
one month and there was no active movement at the shoulder. Transient slight sensory loss over middle third of arm (? sensory branch of circumflex) was noticed in the fourth week. The deltoid started to show wasting, as did the external rotators of the shoulder, elbow flexors and extensors, and to a less extent the forearm muscles, chiefly the extensors, but there was no loss of response to faradism.

Treatment was by massage, radiant heat and supervised movements, and from the sixth week gradual improvement with satisfactory recovery of external rotation and flexion of the forearm occurred. Recovery of full extension at elbow (some biceps contracture) and full true shoulder abduction was delayed until the fourth month. Definite wrist drop was absent throughout.

Was this a paralysis or a pseudo-paralysis? At first a reflex fixation following injury was suggested. The muscle hyperaesthesia, the transient sensory loss, the elbow weakness and the more definite muscle wasting suggested some secondary involvement of nerves. The early and good recovery of the external rotators and the weakness of elbow extension suggest involvement of the posterior cord of the plexus, rather than the nerve roots, but the lesion cannot have been complete, as the carpal extensors were not demonstrably involved.

Case 9. Five years ago a three-year-old boy was admitted with apparently minor injuries sustained in a motor accident while playing on the street. The next day he persisted in refusing to use the left arm, which was radiologically normal and gave no evidence of a shoulder dislocation. He improved slowly when treated for six weeks in an abduction splint and later by supervised exercises.

The left arm now hangs normally from the shoulder, the elbow held in $30^{\circ}$ of flexion, slight wrist drop and ulnar deviation of wrist present. Measurement shows relative shortening of $\frac{3}{4}$ in. in the arm and $\frac{1}{2}$ in. in the forearm. Muscle wasting is slight and confined to triceps and carpal and digital extensors. Though the left hand is slightly smaller than the right, all intrinsic muscles are well developed and acting. Abduction of the arm begins with scapular movement, but only slight relative weakness of true abduction, while external rotation is normal. Full extension of the inter-phalangeal joints is only obtained by flexion of hand at the wrist, while finger flexion is not associated with the normal fixation of the carpus in dorsi-flexion. The triceps shows slight weakness. There is no sensory loss, and apart from diffuse rarefaction at elbow and hand the bones are radiologically normal.

The absence of residual paralysis in the external rotators, elbow flexors, or supinators, the weakness just demonstrable in the deltoid (circumflex nerve) and more marked in the territory of the musculo-spiral nerve-especially of the more distal muscles, is suggestive of a lesion of the posterior cord or the nerves arising from it. In a lesion to the upper nerve roots weakness of the finger extensors should be associated with much more extensive residual weakness. The lesion is incomplete, there is no sensory loss and all muscles have retained some power.

Case 10. Two weeks before first attendance an older child lifted the patient, then an infant of fifteen months, from the ground by the left arm. There appeared to be no pain after the first day and he was treated as a minor injury at another hospital. Treatment for almost one year in an abduction splint and with massage and movements produced only slight improvement. 
Now at the age of seven years he is of poor general nutrition and muscle development, without evidence of spinal cord or sympathetic nerve injury. The left side of the chest is under-developed, the shortened flaccid arm hangs vertically downwards, without rotation, the forearm slightly pronated. Extrinsic scapular muscles are poorly developed without evidence of paralysis. The pectoralis major, the deltoid and spinati show marked wasting (fig. 8). The deltoid just contracts, but cannot fix the flail shoulder joint for abduction by the scapula. Flexion, extension and supination at the elbow are functionally

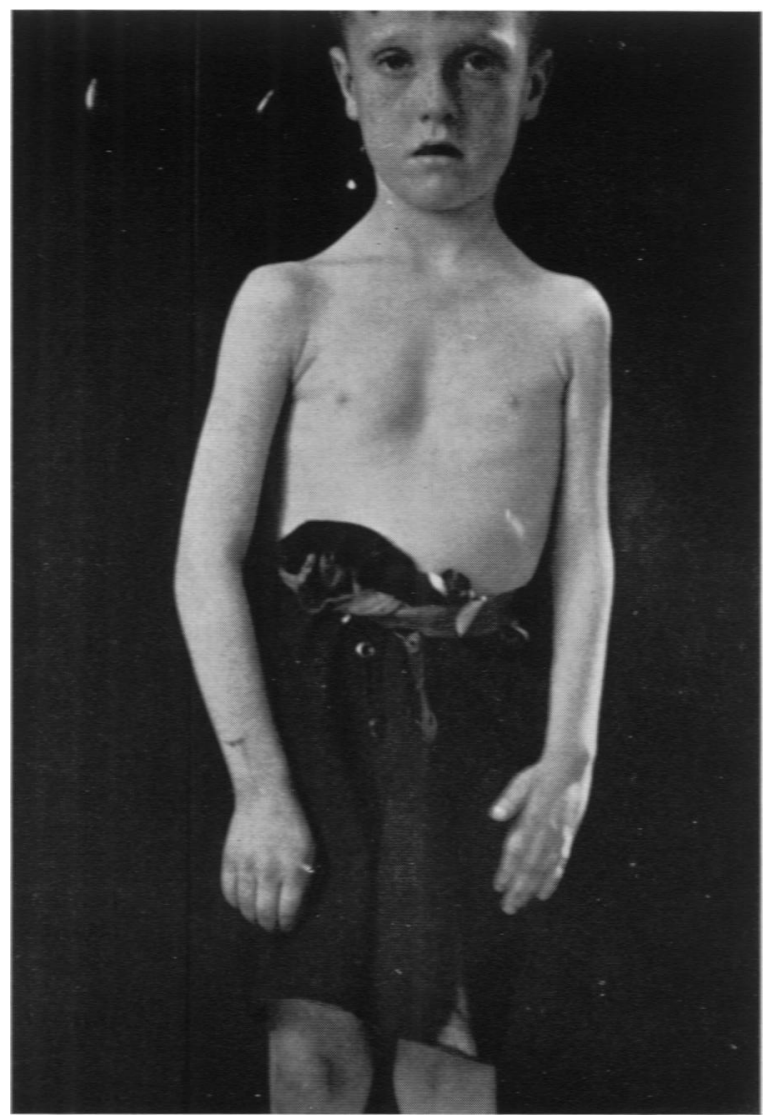

Fig. 8.-Case 10, showing wasting of the limb and sharp shoulder outline in a whole arm lesion.

useless, but there is weak movement of the flexors and extensors of the wrist and fingers and the intrinsic hand muscles are acting.

Radiologically the limb bones show atrophic changes and decalcification only. There is no sensory loss.

The most likely injury is to the roots of the plexus, with less involvement or with better recovery of the lower roots. The extreme wasting and flaccidity suggest anterior poliomyelitis, of which there is no other evidence, although the history is insufficient to exclude this. The trophic changes are those following disuse. 


\section{Etiology}

Obstetrical cases.

INCIDENCE. One patient in slightly over every three thousand children attending the out-patient service of the Belfast Hospital for Sick Children suffered from a birth palsy. The incidence thus appears to be much lower than Duval's figure of one in every two thousand births usually quoted (Thornburn, 1903 ; McFadden, 1928). Fleming (1931) reported twenty-eight cases in eight thousand hospital deliveries. The injury appears to be sustained at birth ; there is nothing to suggest a developmental abnormality. The variable factors are thus : (1) The child ; (2) the mother ; and (3) interference from without -the midwife.

The CHILD. All the cases reviewed have been born by full-time labours, and in many the baby was considered abnormally large. No sex incidence has been observed in any large series. In this series girls were more often affected than boys both in vertex and breech deliveries (seventeen girls to nine boys). An important factor would appear to be the size of the head relative to the shoulders. A large head will, within limits, dilate the passages for the shoulders in a vertex presentation, but will occasion the difficulty in a breech presentation. Of the vertex presentations eight involved the right and seven the left arm, and of eight breech presentations one was bilateral and two involved the left arm. Three transverse lies were dealt with by version late in labour. The high incidence of presentations calling for some interference is significant. In 50 per cent. of cases a definite history of a high degree of cyanotic asphyxia and delay in starting respiration was given. Fairbank (1913) stated that many of his cases were black at birth. This cannot be correlated with the ultimate extent of the lesion. Although cases of phrenic nerve involvement are reported (Kofferath, 1921 ; Epstein, 1927, quoted from Gordon and Forrester Brown, 1933), a special examination of the respiratory system, including screening in two cases, and enquiry as to repeated attacks of pneumonia was negative both in infants with and without asphyxia at birth. A relaxed asphyxiated infant, the product of a difficult labour, is more likely to allow undue separation of hard parts and thus increase the liability of injury of soft parts.

THE MOTHER. The mothers varied from a primigravida of nineteen years to a multipara of forty-one in her eighth pregnancy. The few really long labours were apparently in all cases associated with abnormal presentations. Twelve out of twenty-six cases ( 46 per cent.) were first labours. As the average family in the series was $4 \cdot 3$, this suggests some association with what is generally the most difficult labour. Although two sisters (cases 4 and 7) sustained obstetrical palsies, there was no apparent familial increase of birth injury of any kind, and the mothers do not represent any class of difficult labour case.

INTERFERENCE FROM WITHOUT. Only one labour can be regarded as normal : all the rest appear to have required some assistance, usually quite early in labour, and all had anaesthesia for a short time at least. In the great majority the application of forceps (thirteen out of fifteen vertex cases), version late in labour (three cases), manipulations to bring down an arm in a breech case (one 
case) or great difficulty in delivery of the shoulders (three cases) was described. The association of other injuries in some of these cases, a broken femur in one, bruising of the whole of the affected side, extensive cuts to the face and head (four cases), and probably the increased frequency of sternomastoid tumours are suggestive of unusually difficult labours. In Sever's large series of eleven hundred cases only 5 per cent. were normal labours.

POST-NATAL INJURIES. The varied and often uncertain mechanism of traumatism is illustrated by cases 8,9 and 10 . Williamson (1897) and Vandenbossche (1910) emphasize the great variety of the traumatic force, often slight and almost unnoticed at the moment, such as carrying a weight on the shoulder with the head inclined to the opposite side or a slip while carrying a heavy weight in the hand. One infant of eighteen months had a brachial paralysis because his father had suddenly snatched him up by the armpits (compare case 10). Only occasionally is a dislocation of the shoulder associated even in the adult.

\section{Clinical investigation}

In general this group of twenty-nine children are of average health and nutrition, and appear to lead a fairly normal physical and mental life. One case (case 6) apparently showed a tendency to over-compensate for his disability by noisy, aggressive behaviour with his companions.

Inspection. The appearance of the affected limb varies with the degree o involvement and with the many complex factors in recovery-muscle balance, contractures, muscle wasting and so-called trophic changes. The typical position in the early cases is well known. The deltoid (cervical nerves, fifth and sixth, i.e. C.N. 5-6 ; or C.N. 5 (Harris, 1904)) and the spinati (C.N. 5-6 or C.N. 5, Harris) are paralysed. The sternal head of the pectoralis major (C.N. 8 and Th. 1 or C.N. 6-7-8 (Harris)) and latissimus dorsi (C.N. 6-7-8) escape in great part and cause the characteristic rotation inwards of the arm The elbow is extended, the triceps (C.N. 6-7-8) either escaping altogether or being less involved than the flexors (C.N. 5-6). The supinators of the forearm (biceps and supinator brevis and possibly brachio-radialis supplied by C.N. 5-6 or C.N. 5 (Harris)) are paralysed, as is usually the pronator teres (C.N. 6 or C.N. 5 (Harris)), but the pronator quadratus will produce pronation of the forearm. This with the marked internal rotation of the arm causes the palm of the hand to look backwards and laterally (see fig. 1). The radial flexors of the wrist (C.N. 6 or C.N. 6-7-8, Harris) may be weakened sufficiently to allow ulnar deviation by the flexor carpi ulnaris (C.N. 8, Th. 1). The persistence of some carpal extensor weakness should not be regarded as evidence of a whole arm lesion (compare cases 5, 7,9 and 10).

Improvement will occur in almost all cases, usually starting first in the periphery, probably as the more distal muscles receive a smaller part of their nerve supply from the upper roots. In a number of patients, in whom muscle imbalance has resulted, either from slow or no recovery of paralysed muscles, contractures and often some degree of subluxation of the head of the humerus 
develop, and the limb assumes a different rest position (case 4 and especially case 6). On inspection (fig. 4 and 9) there is slight abduction, usually less than $45^{\circ}$, and marked internal rotation of the humerus. The humerus is slightly flexed forwards (abducted in the antero-posterior plane). The outer part of the scapula may be a little further forward than usual owing to the new position of the limb and probably also to pectoral contracture. The pronated forearm is usually flexed at the elbow, though rarely more than $30^{\circ}$.

Palpation. As Thomas (1916) emphasizes, the humeral head projects

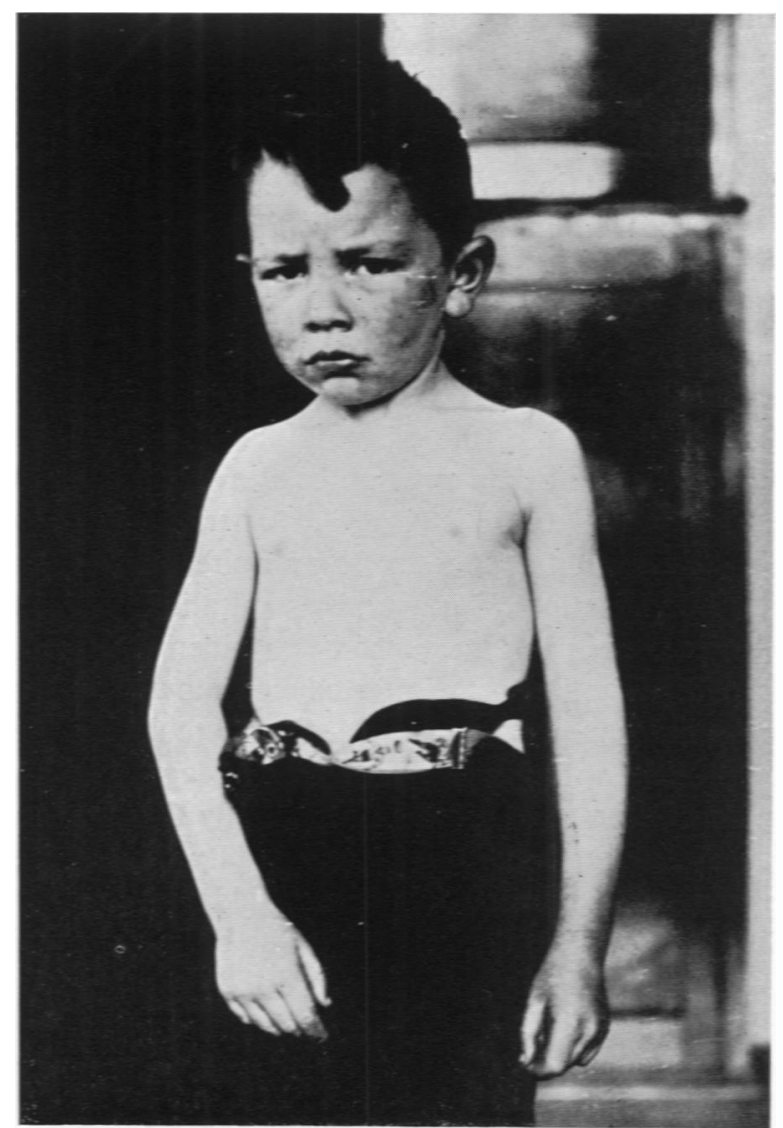

FIG. 9.-Developing contractures less marked than in case 6 (fig. 4).

normally in front of the acromion as much as it does outside it, while there is a depression on pressure of the examining finger under the posterior edge of the acromion. Departure from this, associated in some cases with visible flattening of the front of the shoulder and fullness below the angle of the acromion behind, has been sufficiently marked in six cases to indicate a posterior subluxation. The anterior subluxation described by Bullard (1907) has not been seen. The humeral head probably tends to be driven in whatever direction the muscle support of the joint is most deficient. 
In fifteen of the twenty-seven arms involved in birth injuries there was residual limitation of passive external rotation of the arm. This was associated constantly with contractures demonstrable in the pectoralis major and latissimus dorsi, and possibly of the subscapularis tendon, and in over half of these cases, including all the most marked, full extension at the elbow was also limited. Passive abduction at the shoulder joint can be tested in different positions of the arm, ranging from so-called abduction in the antero-posterior plane to abduction in the coronal (frontal) plane. Abduction in the mid-position (McGregor, 1937) or true scapular plane (Johnston, 1937) is the most important. In the coronal plane full elevation is only attained by external rotation of the humerus (Martin, 1932 ; McGregor, 1937 ; Johnston, 1937). Thus contractures tending to fix the arm in internal rotation may act as an additional limiting factor to abduction. Limitation of passive abduction, confined to those patients showing quite definite limitation of external rotation, was always associated with contractures, often marked in the latissimus dorsi and teres major. Contractures of the inferior gleno-humeral ligament (McFadden, 1928) and elongation of the acromion process (Sever, 1920) are probably not primary causes.

Active movements. Although it is easy to determine that a joint moves, it is by no means easy to determine beyond doubt what agent causes its movement. This necessitates a study of the rôle of the different muscles in carrying out movements, and a realization that muscles, as Beevor (1903) has emphasized, have no independent or isolated existence. The nervous mechanisms will use any agent capable of carrying out the motion required. The optimal position for contraction, the elimination of gravity, the limitations of contractures, and the fixation of the proximal parts must also be considered.

In two patients the scapula of the affected side showed 'winging,' and this prominence became more marked as the patient tried to abduct the arm towards the horizontal or pushed with the outstretched limb against a resisting object. In itself prominence of the scapula with the arms by the side is without significance and is usually bilateral. In view of the difficulty of adequate examination in the presence of other paralysis about the shoulder, a difficulty appreciated by Duchenne (1872), it appears likely that the site of injury may in some cases involve the nerve roots proximal to the origin of the long thoracic nerve or that the nerve may be involved in its course behind the plexus by secondary scar tissue. Bullard (1907) almost alone affirms that the serratus magnus is usually involved, but only refers to ' winging' of the scapula. In all cases failing to show complete recovery, with some residual deltoid weakness or a joint fixed by contractures, the inferior angle of the scapula, if free, began to move outwards from the beginning of abduction. This movement is to be distinguished from the slight gliding motion emphasized by Lockhart (1930) as occasionally marking the beginning of arm abduction, which normally proceeds for almost $45^{\circ}$ before the scapula commences to move.

In almost all cases of birth paralysis the loss of the power of true external rotation is more marked than that of true abduction. If the scapula is free this is even more fully emphasized, and it is this, often variably developed, scapular mobility which renders such functional tests as attempting to touch the nose, 
eyebrows, the back of the head or the vertebral spines of uncertain significance in examining true shoulder joint movements. The limitation and weakness of supination at the elbow, often imposed by contractures, further increase the functional disability of internal rotation of the arm (fig. 5). Only in the patients with involvement of the whole arm is there any permanent functionally significant weakness of flexion and extension at the elbow.

Owing to 'trick' movements of the fingers by the long forearm muscles (Wood Jones, 1920), paralysis of the intrinsic hand muscles is difficult to exclude. While there may be general wasting, possibly from disuse, some of the muscles may be seen or felt to contract. In root lesions paralysis confined to these muscles with tendency to develop a claw hand should only occur in lesions of the first thoracic root (Klumpke's paralysis). In whole arm lesions the wider extent of the lesion rarely permits gross imbalance.

Sympathetic lesions. Anatomically pupillary inequality and other evidence of sympathetic involvement should only be encountered in lower or whole arm lesions. Sever (1920) finds it 'in a good many cases' and not apparently confined to whole arm lesions. It was absent in patients with involvement of the whole arm (cases 7 and 10) and in a patient with early lower arm lesion seen since the rest of the series. Its absence does not at all exclude injury to the lower roots and its presence is inconstant (Vandenbossche, 1910).

Sternomastoid tumours. Sternomastoid tumours occurred in seven cases (27 per cent.), in two involving the opposite side to the affected arm. The association is mentioned in Middleton's paper (1930) on sternomastoid tumours ; he found eleven out of fifty-four case records of Erb's paralysis showed the presence of such tumour.

Trophic changes. Changes in growth and nutrition of the limb have been but little studied and generally dismissed as ' neurotrophic' (Frauenthal, 1912 ; Sever, 1920). In almost all patients showing residual paresis there is shortening of the limb bones, affecting the arm and forearm. In one case, associated with some weakness of carpal extensors and disuse of the forearm, this was most marked in the forearm (case 5, fig. 2 and 3). In severe cases with a practically useless limb the clavicle shows marked shortening, up to 1 in., on the affected side. The scapula and indeed the chest of the affected side may be underdeveloped (cases 7 and 10, fig. 7 and 8). In a few cases the unaffected limb may show compensatory hypertrophy. Skiagrams show the bones to be decalcified and thinner than on the normal side with sometimes the later appearance of ossific centres. Over an analgesic area in case 7 the skin was thin, shiny and devoid of hairs ; in this and in the hands of two other cases (case 5) associated with disuse there was a deficient development of the natural skin creases, but the pulp of the fingers and the nails were not abnormal. There is no evidence that the sympathetic exerts any influence on growth or muscle function. All changes seen are such that disuse and diminished blood supply would explain. Few trophic changes can be regarded as due to the withdrawal of the direct nutritional influence of nerve cells on affected cells (Lewis, 1936).

Sensation. In eight cases dependable observations on sensation were possible. In case 7 an equal loss of sensation to pin-prick and light touch 
suggests comparison with those cases showing similar type of loss following division of the dorsal roots or the roots of the plexus (Head and Sherren, 1905). The flare response (Lewis, 1927) over this area was positive, suggesting a lesion proximal to the posterior root ganglion. Two upper arm birth palsies showed sensory loss over the lateral aspect of the arm-almost over the deltoid insertion. Sensory loss has been described in the adult traumatic cases (Williamson, 1897), but is stated to be usually transient (Warrington and Jones, 1906). The fifth and possibly the sixth cervical root can be divided without sensory loss (Sherren, 1906).

There was no correlation in the upper arm lesions between residual sensory loss and extent of motor paralysis. Pollock (1934) in a detailed investigation, found no specific relation between the return of sensation in individual nerve fields and motor recovery in muscles deriving their nerve supply from the same mixed nerves. The most marked bony changes were associated with absolutely normal sensation (case 6), and joints radiologically normal with sensory loss while contractures developed in the presence of, or without, sensory loss.

Radiography. Certain changes are described (Sever, 1920, 1925; Gilmour, 1925 ; Jepson, 1930 ; McMurray, 1937), which, however, are of no diagnostic value. The humerus may be somewhat atrophic with alteration in trabeculae, the glenoid flattened and with arthritic changes, the coracoid elongated and the acromion show hooking or 'beak' formation (McMurray, 1937). The most definite change, though seen but twice in well-marked cases of subluxation, is the development of a cone-shaped humeral head, flattened side next the axilla (case 6, fig. 6). Cervical ribs were present in one case (case 4) and the cervical lateral processes of the seventh cervical vertebra were elongated in another (case 7). While x-rays may not always reveal the presence of cervical ribs (Southam and Bythell, 1924) there is no significant demonstrable evidence of a high incidence of prefixation of the brachial plexus. Radiologically there was no evidence of any epiphyseal lesion. In cases showing deficient full extension at the elbow this was not associated with the demonstrable bony changes found by Bullard (1907).

Electrical reactions. Only in one case (case 8) were these employed. A general anaesthetic is required in young children for accurate localization of response (Fairbank, 1913) and the results are often misleading in prognosis (Froelich, 1921). The volitional test scientifically employed will give as much information as the equally difficult electric tests, and greater help with the more important question of the muscle balance of joints.

\section{Pathology}

There is considerable operative data (Kennedy, 1903, 1904 ; Taylor, 1921 ; Boorstein, 1923) to support the involvement in upper arm cases of the fifth and sixth roots, or sometimes the fifth alone (Sherren, 1906; Fairbank, 1913) proximal to the plexus. At least half the cases showing residual weakness appear to require a more extensive lesion than the avulsion of the supraclavicular nerve suggested by McFadden (1928). Boyer (1911-12) reports an 
autopsy on a long-standing case in which definite changes in the antero-lateral region of the cord were found, and Sever (1925) claims to find cord injury in a ' noticeable number' of cases. Every case was examined for evidence of residual lesion and questioned for significant delay in learning to walk with an entirely negative result.

Lesions may be produced in the cadaver illustrating that the effect of forcible traction, pulling the head to the opposite side, or rotation of the head is maximal on the upper roots of the plexus, owing chiefly to their more acutely downward course, and that the roots tear before the plexus or the branches (Sever, 1920 ; McFadden, 1928). These can only give information as to a possible mode of production of what is often a severe grade of lesion and make no recognition of the effect of pressure from haemorrhage or scar tissue formation following laceration of the deep cervical fascia. Those patients making a complete recovery (e.g. case 2) show such rapid improvement that in them pressure from blood or oedema of neighbouring parts seems probable. Direct pressure from forceps should only produce the lesion if associated with gross damage to superficial structures. Traumatism from the clavicle is considered by Williamson (1897) and Derganz (1930). Kustner in 1889 and Peltesohn in 1914 (Froelich, 1921) suggested a fracture of the upper extremity of the humerus with secondary involvement of nerves. Shortening is better explained by disuse than by such co-called epiphyseal injury (case 5, fig. 2 and 3 ).

T. Turner Thomas $(1911,1914,1916)$ from a study of shoulder-joint injuries in adults advanced the view that laceration of the axillary portion of the capsule, following sprains and dislocations, and the resultant extravasation of blood, lymph and synovial fluid set up a non-infective inflammation around the branches of the brachial plexus. In obstetric cases he regards the subluxation as primary and resulting from direct pressure by the maternal pelvis. This view is strongly opposed by Taylor (1921). In cases of obstetrical palsy the presence of demonstrable displacements at the shoulder joint only in cases of some duration, the existence of intermediate lesions apparently connecting such subluxation with the slow development of contractures at the shoulder and often also at the elbow has given no support to this view. In cases 8 and 9 'sprains' of the shoulder joint must be considered in the etiology of lesions of a somewhat different nature. In the latter there is no involvement of the external rotators : the territory of the muscle paralysis is practically that of the posterior cord. In the former the arm is rendered motionless, painful, resisting passive movement, with paralysis more extensive than severe in any one muscle group, with muscle wasting, transitory slight sensory loss, muscle hyperaesthesia, associated with rapid recovery, in particular of the external rotators, residual weakness being mainly in the elbow extensors. This patient was closely observed over a prolonged period, and at all stages appeared to differ materially from the picture presented by any birth palsy.

\section{Diagnosis}

A careful history and some patience in the examination will usually lead to a diagnosis in the birth palsies. In a few cases seen late, and especially in the post- 
natal traumatic lesions, the localization of the lesion and even the nature of the lesion may be decided only in terms of probability. A realization of the different picture presented at different stages, a detailed correlation of muscle weakness with possible injury in nerve trunks, nerve roots, or anterior horn cells, with an appreciation of the individual variations, is essential.

The arm may be held motionless and in the typical position from reflex guarding in any painful condition about the shoulder, and for Thomas, Peltesohn and others this pseudo-paralysis is the primary cause of the rest position of the limb. A limp, flaccid limb, usually painless on movement, immediately after birth will almost exclude epiphyseal separation or primary subluxation of the shoulder-if, indeed, these ever occur. Birth fractures, occasionally showing temporary wrist drop later from involvement of the musculo-spiral nerve, may be considered. Syphilitic epiphysitis, the pseudo-paralysis of scurvy, early osteomyelitis or tuberculosis of the shoulder joint in the slightly older child can be excluded. Occasionally children recently lifted by the arm or shoulder are seen who have since ceased to use the arm. Passive movement is usually free and painless ; active movement may appear absent for some hours or even overnight, though often careful examination reveals some power in all the muscle groups (compare case 10).

Of the spastic or cerebral palsies a monoplegia is the rarest. Evidence of hemiplegia or diplegia, mental and developmental arrest and the preservation or return of clumsy, coarse movement at the proximal joints, while the hand movements are more affected will be helpful. Anterior poliomyelitis may cause real difficulty in the lesions of older children, the febrile period and the transient involvement of other muscles escaping the notice of the parent. In cases studied with residual arm involvement the incidence on the deltoid was marked, but external rotation was proportionately less involved. In uncomplicated root lesions the external rotators appear to be the last muscles to recover. The possibility of pressure on the spinal cord or roots by spinal caries, tumours, syringomyelia or cervical rib (lower arm, or Klumpke type of lesion only simulated) may be considered. Behaviour in nerve injuries does not suggest the more constant pain of pressure lesions. Adequate examination will exclude infantile muscular atrophy of spinal origin, the myopathies or functional disease in older children.

\section{Prognosis}

Trainor and Crothers (1923) from Sever's clinic consider that full recovery is exceptional. Fleming (1931) obtained recovery in the great majority of his cases (twenty-one out of twenty-eight cases). Though a completely useless arm is rare, there appears to be no guide but the progress of the case. Provided contractures are prevented every patient will improve-often for years. In this group of twenty-six cases involving twenty-seven arms (one bilateral) six made a complete recovery. This recovery was complete or all but complete within three months, a finding supported by Boorstein (1923). In five examples of incomplete recovery function was chiefly limited by weakness of the external rotators, the hand being brought to the mouth or forehead in a useful position, 
deltoid action being full or almost full. Six arms showed a varying degree of posterior subluxation with proportionate degree of development of firm contractures and limitation of movement. One virtually untreated patient with whole arm involvement although showing no subluxation has functionally almost a useless limb. In the remaining nine arms, including two still under treatment, contractures have failed to cause definite subluxation, but limit external rotation and abduction so that in only a few can the hand be raised above the level of the chin. In hospital a number of late cases which have failed to improve may be seen. However, all but five patients (six paralysed arms) attended before the age of three months, and, of these, all but two before the age of two months. Omitting late cases there are six complete recoveries in twenty-one cases ( 29 per cent.), and a good functional result in four (19 per cent.), while only two treated cases show subluxation. Reference to the case notes (cases 8, 9 and 10) will show how difficult and guarded prognosis must be in post-natal traumatic cases.

\section{Treatment}

Complete recovery has followed inadequate treatment, and might follow no treatment in many cases recovering within three months. The justification for energetic and painstaking treatment is the relatively better function and freedom from contractures in the thoroughly treated patients. The paralysed muscles must be placed in a position in which they are relaxed beyond their normal position of equilibrium. A splint (Fairbank, 1913 ; Platt, 1921 ; Boorstein, 1923) may be used to hold the arm in right-angled abduction and should maintain it well braced back to prevent pectoral contractures (Mackenzie, 1930), and with the forearm in right-angled flexion and pronation, correcting wrist drop or carpal deviation if present. A collar and cuff fixing the dorsum of the hand to the back of the head has given adequate fixation in eight out of ten early cases in which it was employed. With intelligent co-operation it is especially useful in such cases. McMurray (1937) advises this method in the young infant.

The use of movements, often associated with music, passive at first and later active, has been advocated, splints being used only for developing contractures (Trainor and Crothers, 1923 ; Sever, 1925 ; Gordon and Forrester Brown, 1933). Education of true shoulder muscles is difficult owing to free scapular movement. It seems better that early fixation should prevent contractures. Supervised movements are of great value, but may supplement rather than replace fixation in the practice of most clinics. Electricity appears to have little place in treatment; indeed Boorstein (1924) describes its use as cruel.

The subluxation may be treated by forcible manipulation on one or more occasions and prolonged fixation in plaster (Whitman, 1905; Thomas, 1914, 1916). Platt (1921) and Gilmour (1925) think this only suitable for infants and young children. In the open operation (Fairbank, 1913 ; Sever, 1920, 1925 ; Taylor, 1921 ; Boorstein, 1923 ; McMurray, 1937) the subscapularis and occasionally the pectoralis major tendons are cut to allow full rotation and abduction of the humerus. Some range of movement at the shoulder joint 
should be present and the joint free from arthritic change before operation. In two cases the subscapularis tendon was divided before the age of six months. Now five years later in both cases the joint has an almost full range of passive movement, is rather lax, active external rotation is limited and functionally of reduced value owing to weakness, while the true shoulder abductors are acting very little (fig. 10). In another case two operations after the age of six years have failed to prevent internal rotation, owing to the inadequate recovery of

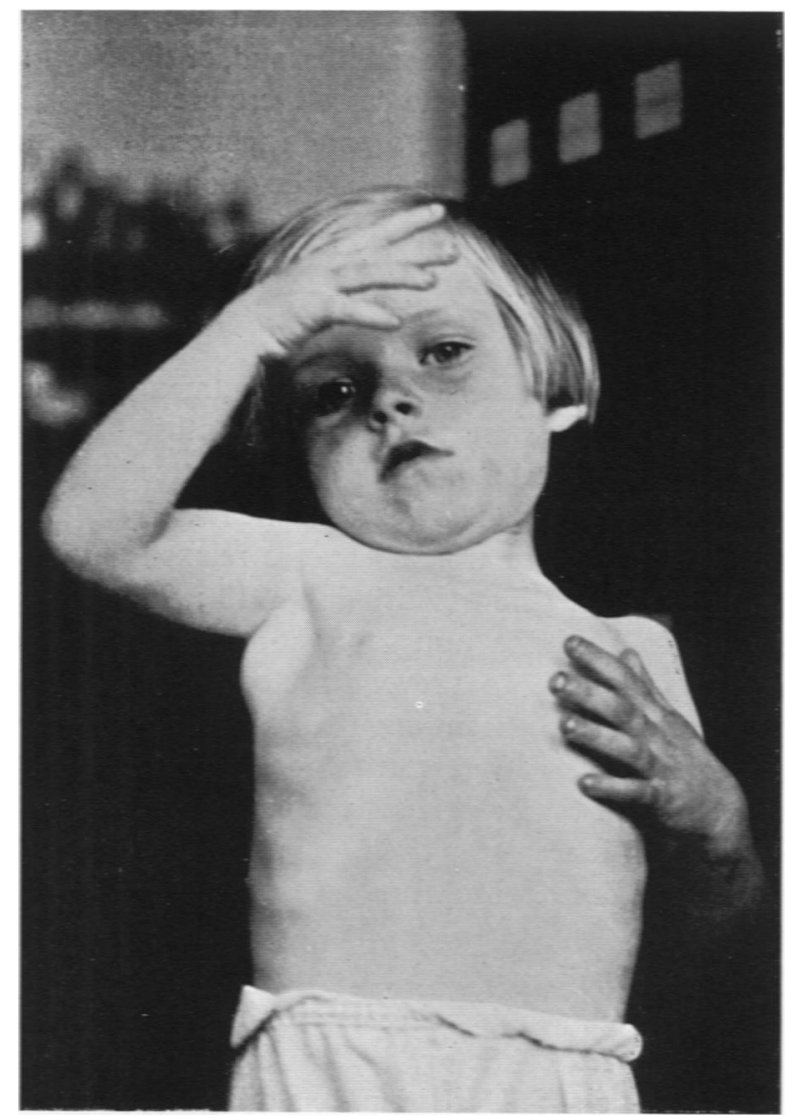

Fig. 10.-Subscapularis tenotomy, but function still limited owing to persistent muscle weakness.

the external rotators, the possible extent of which cannot be predicted before operation. The variety of tendon grafting operations is evidence of their general uselessness. Suggested bone operations include correction of internal rotation by a humeral osteotomy, and ankylosis for a flail shoulder joint, but only if elbow movement is satisfactory. Taylor (1921), who has had a considerable experience of operations directed to the damaged nerves, now prefers to wait as long as any improvement is taking place. In the hands of a few such operations are perhaps justified in patients with whole arm lesions showing no recovery. 
The treatment of post-natal traumatic injuries is directed on similar lines to that of birth palsies. A metal abduction splint will be required, and more active co-operation may be hoped for from the patient.

\section{Summary}

The progress over a number of years of an unselected group of cases with birth and post-natal brachial nerve palsies has been reviewed and an attempt made to describe and correlate the changes with the possible site of traumatism.

Although the distribution of paralysis in birth palsies supports in general a rupture of the nerve roots from forcible overstretching a variable picture is presented. Two post-natal injuries depart so much from any such picture as to suggest the involvement of the posterior cord or the nerves derived from it, possibly by organization of an exudate from a traumatized shoulder joint.

In the etiology of birth palsies the one almost constant factor is interference from without. Other factors are important as they increase the necessity for this.

Slightly less than one-third of the birth palsies made a complete recovery, and this occurred within the first three months.

Emphasis throughout, especially in treatment, should be on the muscle balance of joints, best determined by a study of voluntary movements, and on the prevention of contractures.

Thanks are due to the Honorary Visiting Staff of the Belfast Hospital for Sick Children for permission to examine patients and case notes, to the Trustees of the Davis Scholarship, to Professor J. H. Biggart for valued help and advice and to Dr. F. M. B. Allen for kind encouragement during the preparation of this manuscript.

\section{REFERENCES}

Ashhurst, A. P. C. (1918). Ann. Surg., 67, 25.

Beevor, C. E. (1903). Lancet, 1, 1,715, 1,783.

Boorstein, S. W. (1923). J. Bone Joint Surg., 5, 778.

- (1924). J. Amer. med. Ass., 82, 862.

Boyer, G. F. (1911-12). Proc. roy. Soc. Med., 5, 31.

Bullard, W. N. (1907). Amer. J. med. Sci., 134, 93.

Delbet, Pierre, and Cauchoix, A. (1910). Rev. Chir., 41, 328, 667.

Derganz, F. (1930). Arch. f. klin. Chir., 160, 169.

Duchenne, G. B. (1861). De l'Electrisation Localisée, second edition, Paris, 348. (1872). Ibid., third edition, 353, 943.

Fairbank, H. A. T. (1913). Lancet, 1, 1,217.

Fleming, G. B. (1931). Brit. med. J., 2, 481.

Frauenthal, H. W. (1912). Amer. J. Obstet., 65, 538.

Froelich, P. (1921). Rev. Chir., 59, 419.

Gilmour, J. (1925). Lancet, 2, 696.

Gordon, R. G., and Forrester Brown, M. (1933). Paralysis in Children, London.

Harris, W. (1904). J. Anat. Physiol., 38, 399.

Head, H., and Sherren, J. (1905). Brain, 28, 116.

Jepson, P. N. (1930). Ann. Surg., 91, 724.

Johnston, T. B. (1937). Brit. J. Surg., 25, 252.

Jones, F. W. (1920). J. Anat., 54, 41. 
Kennedy, R. (1903). Brit. med. J., 1, 298. (1904). Ibid., 2, 1,065.

Lewis, T. (1927). The Blood Vessels of the Human Skin and Their Responses, London. - (1936). Vascular Disorders of the Limbs, London.

Lockhart, R. D. (1930). J. Anat., 64, 288.

McFadden, G. D. F. (1928). J. Bone Joint Surg., 10, 661.

McGregor, L. (1937). Brit. J. Surg., 24, 425.

Mackenzie, Colin (1930). The Action of Muscles, London, 79.

McMurray, T. P. (1937). Practice of Orthopaedic Surgery, London.

Martin, C. P. (1932). Brit. J. Surg., 20, 61.

Middleton, D. S. (1930). Brit. J. Surg., 18, 188.

Platt, H. (1921). Brit. med. J., 2, 885.

Pollock, L. J. (1934). Surg., Gynec. \& Obstet., 59, 858.

Sever, J. W. (1920). Canad. med. Ass. J., 10, 141.

- (1925). J. Amer. med. Ass., 85, 1,862.

Sherren, J. (1906). Lancet, 1, 727, 809, 886.

Southam, A. H., and Bythell, W. J. S. (1924). Brit. med. J., 2, 844.

Taylor, A. S. (1921). Ann. Surg., 74, 368.

Thomas, T. T. (1911). Ibid., 53, 77.

(1914). Ibid., 59, 197.

(1916). Amer. J. Obstet. Dis. Wom., 73, 577.

Thornburn, W. (1903). J. Obstet. Gynaec., 3, 454.

Trainor, Mary E., and Crothers, B. (1923). Med. Clin. N. Amer., 7, 979.

Warrington, W. B., and Jones, R. (1906). Lancet, 2, 1,644.

Whitman, R. (1905). Ann. Surg., 42, 110.

Williamson, R. T. (1897). Lancet, 2, 1,522.

Vandenbossche, (1910). Rev. Chir., 41, 819. 